\title{
HYPONORMALITY OF TOEPLITZ OPERATORS WITH RATIONAL SYMBOLS
}

\section{In Sung Hwang ${ }^{1}$ and Woo Young Lee $^{2}$}

1. Department of Mathematics, Institute of Basic Science, Sungkyunkwan University, Suwon 440-746, Korea

(e-mail: ihwang@skku.edu)

2. Department of Mathematics, Seoul National University, Seoul 151-742, Korea

(e-mail: wylee@math.snu.ac.kr)

Mathematics Subject Classification (2000): Primary 47B20, 47B35

\begin{abstract}
In this article we introduce a notion of 'division' for rational functions and then give a criterion for hyponormality of $T_{\bar{g}+f}(f, g$ are rational functions) in the cases where $g$ divides $f$. Furthermore we show that we may assume, without loss of generality, that $g$ divides $f$ when we consider the hyponormality of $T_{\bar{g}+f}$.
\end{abstract}

\section{Introduction}

A bounded linear operator $A$ on a Hilbert space $\mathfrak{H}$ is said to be hyponormal if its selfcommutator $\left[A^{*}, A\right]=A^{*} A-A A^{*}$ is positive semidefinite. Recall that given $\varphi \in L^{\infty}(\mathbb{T})$, the Toeplitz operator with symbol $\varphi$ is the operator $T_{\varphi}$ on the Hardy space $H^{2}(\mathbb{T})$ of the unit circle $\mathbb{T}=\partial \mathbb{D}$ in the complex plane $\mathbb{C}$ defined by

$$
T_{\varphi} f=P(\varphi \cdot f)
$$

where $f \in H^{2}(\mathbb{T})$ and $P$ denotes the orthogonal projection that maps $L^{2}(\mathbb{T})$ onto $H^{2}(\mathbb{T})$. Normal Toeplitz operators were characterized by a property of their symbols in the early 1960's by A. Brown and P. Halmos [BH]. The problem of determining which symbols induce hyponormal Toeplitz operators was completely solved by C. Cowen [Co] in 1988. Here we shall employ an equivalent variant of Cowen's theorem that was proposed by T. Nakazi and K. Takahashi in $[\mathrm{NT}]$.

Cowen's theorem. ([Co], $[\mathrm{NT}])$ Suppose that $\varphi \in L^{\infty}(\mathbb{T})$ is arbitrary and put

$$
\mathcal{E}(\varphi):=\left\{k \in H^{\infty}(\mathbb{T}):\|k\|_{\infty} \leq 1 \text { and } \varphi-k \bar{\varphi} \in H^{\infty}(\mathbb{T})\right\}
$$

\footnotetext{
${ }^{1}$ Supported in part by a grant from Faculty Research Fund, Sungkyunkwan University, 2004.

${ }^{2}$ Supported in part by a grant (R14-2003-006-01000-0) from the Korea Research Foundation.
} 
Then $T_{\varphi}$ is hyponormal if and only if the set $\mathcal{E}(\varphi)$ is nonempty.

Cowen's method is to recast the operator-theoretic problem of hyponormality for Toeplitz operators into the problem of finding a solution of a certain functional equation involving its symbol.

A function $\varphi \in L^{\infty}$ is said to be of bounded type (or in the Nevanlinna class) if there are functions $\psi_{1}, \psi_{2}$ in $H^{\infty}(\mathbb{D})$ such that

$$
\varphi(z)=\frac{\psi_{1}(z)}{\psi_{2}(z)}
$$

for almost all $z$ in $\mathbb{T}$. Evidently, rational functions in $L^{\infty}$ are of bounded type.

For an inner function $\theta$, write

$$
\mathcal{H}(\theta):=H^{2} \ominus \theta H^{2}
$$

Note that ker $H_{\bar{\theta}}=\theta H^{2}$ and $\operatorname{ran} H_{\bar{\theta}}^{*}=\mathcal{H}(\theta)$. It was shown [Ab, Lemma 6] that if $T_{\varphi}$ is hyponormal and $\varphi$ is not in $H^{\infty}$ then

$$
\varphi \text { is of bounded type } \Longleftrightarrow \bar{\varphi} \text { is of bounded type. }
$$

In $[\mathrm{Ab}]$, it was also shown that

$$
\varphi \text { is of bounded type } \Longleftrightarrow \operatorname{ker} H_{\varphi} \neq\{0\} \Longleftrightarrow \varphi=\bar{\theta} b \text {, }
$$

where $b \in H^{\infty}$ and $\theta$ is an inner function such that the inner parts of $b$ and $\theta$ are relatively prime. Therefore we can see (cf. [GS], [Gu2]) that if $\varphi=\bar{g}+f\left(f, g \in H^{2}\right)$ is of bounded type and $T_{\varphi}$ is hyponormal then we can write

$$
f=\theta_{1} \theta_{2} \bar{a} \quad \text { and } \quad g=\theta_{1} \bar{b}
$$

for some inner functions $\theta_{1}$ and $\theta_{2}$, where $a \in \mathcal{H}\left(\theta_{1} \theta_{2}\right)$ and $b \in \mathcal{H}\left(\theta_{1}\right)$. Here we assume that the inner parts of $a$ and $\theta_{1} \theta_{2}$ are relatively prime and also the inner parts of $b$ and $\theta_{1}$ are relatively prime.

Let $\theta$ be a finite Blaschke product of degree $d$. We can write

$$
\theta=e^{i \xi} \prod_{i=1}^{n} B_{i}^{n_{i}},
$$

where $B_{i}(z):=\frac{z-\alpha_{i}}{1-\overline{\alpha_{i}} z},\left(\left|\alpha_{i}\right|<1\right), n_{i} \geq 1$ and $\sum_{i=1}^{n} n_{i}=d$. Let $\theta=e^{i \xi} \prod_{j=1}^{d} B_{j}$ and each zero of $\theta$ be repeated according to its multiplicity. Note that this Blaschke product is precisely the same Blaschke product in (1). Let

$$
\phi_{j}:=\frac{d_{j}}{1-\overline{\alpha_{j}} z} B_{j-1} B_{j-2} \cdots B_{1} \quad(1 \leq j \leq d)
$$

where $\phi_{1}:=d_{1}\left(1-\overline{\alpha_{1}} z\right)^{-1}$ and $d_{j}:=\left(1-\left|\alpha_{j}\right|^{2}\right)^{\frac{1}{2}}$. It is well known that $\left\{\phi_{j}\right\}_{j=1}^{d}$ forms an orthonormal basis for $\mathcal{H}(\theta)$ (cf. [FF, Theorem X.1.5]). 
Let $f \in H^{\infty}$ be a rational function such that $f(0)=0$. Then we may write

$$
f=p_{m}(z)+\sum_{i=1}^{n} \sum_{j=0}^{l_{i}-1} \frac{a_{i j}}{\left(1-\overline{\alpha_{i}} z\right)^{l_{i}-j}} \quad\left(0<\left|\alpha_{i}\right|<1\right)
$$

where $p_{m}(z)$ denotes a polynomial of degree $m$. Let

$$
\theta=z^{m} \prod_{i=1}^{n} B_{i}^{l_{i}}
$$

where $B_{i}(z):=\frac{z-\alpha_{i}}{1-\overline{\alpha_{i}} z}$. Observe that

$$
\frac{a_{i j}}{1-\overline{\alpha_{i}} z}=\frac{\overline{\alpha_{i}} a_{i j}}{1-\left|\alpha_{i}\right|^{2}}\left(\frac{z-\alpha_{i}}{1-\overline{\alpha_{i}} z}+\frac{1}{\overline{\alpha_{i}}}\right)
$$

Letting $a:=\theta \bar{f}$, we can see that $a \in \mathcal{H}(\theta)$ and $f=\theta \bar{a}$. Thus if $\varphi=\bar{g}+f \in L^{\infty}$, where $f$ and $g$ are rational functions with $f(0)=g(0)=0$ and if $T_{\varphi}$ is hyponormal, then we can write

$$
f=\theta_{1} \theta_{2} \bar{a}, \quad g=\theta_{1} \bar{b}
$$

for some finite Blaschke products $\theta_{1}, \theta_{2}$ and $a \in \mathcal{H}\left(\theta_{1} \theta_{2}\right)$ and $b \in \mathcal{H}\left(\theta_{1}\right)$, where the inner parts of $a$ and $\theta_{1} \theta_{2}$ are relatively prime and the inner parts of $b$ and $\theta_{1}$ are relatively prime.

It was shown in [Zhu] that the hyponormality of $T_{\varphi}$ with polynomial symbols $\varphi$ can be reduced to a Carathéodory-Schur interpolation problem (also see [HL] for another criterion). By comparison, it was observed in [Gu1] that the hyponormality of $T_{\varphi}$ with rational symbols $\varphi$ can be reduced to a tangential Hermite-Fejér interpolation problem. In this article we define the division $\frac{f}{g}$ for rational functions $f, g$ and present a criterion for hyponormality of $T_{\bar{g}+f}$ when $g$ divides $f$, where $f, g$ are rational functions. Furthermore we show that the condition " $g$ divides $f$ " can be assumed without loss of generality when we study the hyponormality of $T_{\bar{g}+f}$.

\section{Main Results}

We need several auxiliary lemmas to understand the main results.

Lemma 1. If $\theta_{1}$ is a Blaschke product and $\theta_{2}$ is an inner function then

$$
\mathcal{H}\left(\theta_{1} \theta_{2}\right) \subset \mathcal{H}\left(\theta_{1}\right) \cdot \mathcal{H}\left(z \theta_{2}\right)
$$

In particular, if $\theta_{1}$ and $\theta_{2}$ are finite Blaschke products then

$$
\mathcal{H}\left(\theta_{1} \theta_{2}\right)=\mathcal{H}\left(\theta_{1}\right) \cdot \mathcal{H}\left(z \theta_{2}\right)
$$

Proof. We first observe that for any inner functions $\theta_{1}$ and $\theta_{2}$,

$$
\mathcal{H}\left(\theta_{1} \theta_{2}\right)=\theta_{2} \mathcal{H}\left(\theta_{1}\right)+\mathcal{H}\left(\theta_{2}\right)
$$


and hence

$$
\mathcal{H}\left(\theta_{1}\right) \cdot \mathcal{H}\left(z \theta_{2}\right)=\mathcal{H}\left(\theta_{1}\right) \cdot\left[\theta_{2} \mathcal{H}(z)+\mathcal{H}\left(\theta_{2}\right)\right]=\theta_{2} \mathcal{H}\left(\theta_{1}\right)+\mathcal{H}\left(\theta_{1}\right) \cdot \mathcal{H}\left(\theta_{2}\right)
$$

We now claim that if $\theta_{1}$ is a Blaschke product then

$$
\mathcal{H}\left(\theta_{2}\right) \subset \mathcal{H}\left(\theta_{1}\right) \cdot \mathcal{H}\left(z \theta_{2}\right)
$$

Towards (5), let $\theta_{1}$ be a Blaschke product of degree $N$ (possibly, infinite). Then we can write

$$
\theta_{1}=e^{i \xi_{1}} \prod_{i=1}^{N} B_{i},
$$

where $B_{i}(z)=\frac{z-\alpha_{i}}{1-\overline{\alpha_{i}} z}\left(\left|\alpha_{i}\right|<1\right)$. We write $H_{0}^{2}:=\left\{z f: f \in H^{2}\right\}$ and $\overline{\mathcal{L}}:=\{\bar{f}: f \in \mathcal{L}\}$ for $\mathcal{L} \subset L^{2}(\mathbb{T})$. Then for any inner function $\theta$,

$$
\mathcal{H}(\theta)=\left\{f \in H^{2}: \bar{\theta} f \in \overline{H_{0}^{2}}\right\}
$$

Suppose $f \in \mathcal{H}\left(\theta_{2}\right)$. Then we have that

$$
\begin{aligned}
\left(1-\overline{\alpha_{1}} z\right) f \in \mathcal{H}\left(z \theta_{2}\right) & \Longleftrightarrow \bar{z} \overline{\theta_{2}}\left(1-\overline{\alpha_{1}} z\right) f \in \overline{H_{0}^{2}} \\
& \Longleftrightarrow \bar{z} \overline{\theta_{2}} f-\overline{\theta_{2}} \overline{\alpha_{1}} f \in \overline{H_{0}^{2}},
\end{aligned}
$$

which implies that $f \in \mathcal{H}\left(\theta_{1}\right) \cdot \mathcal{H}\left(z \theta_{2}\right)$. This proves (5). Hence from (5), we have that

$$
\mathcal{H}\left(\theta_{2}\right) \subset \mathcal{H}\left(\theta_{1}\right) \cdot \mathcal{H}\left(z \theta_{2}\right)=\theta_{2} \mathcal{H}\left(\theta_{1}\right)+\mathcal{H}\left(\theta_{1}\right) \cdot \mathcal{H}\left(\theta_{2}\right)
$$

which implies that $\mathcal{H}\left(\theta_{1} \theta_{2}\right) \subset \mathcal{H}\left(\theta_{1}\right) \cdot \mathcal{H}\left(z \theta_{2}\right)$. This proves $(3)$.

Further if $\theta_{1}$ and $\theta_{2}$ are finite Blaschke products then by $(6), \mathcal{H}\left(\theta_{1}\right) \cdot \mathcal{H}\left(z \theta_{2}\right) \subset \mathcal{H}\left(\theta_{1} \theta_{2}\right)$, which together with (3) proves (4).

The inclusion (3) of Lemma 1 need not hold if $\theta_{1}$ is a singular inner function even though $\theta_{2}$ is a finite Blaschke product. For example, if $\theta_{1}=e^{\frac{z+1}{z-1}}$ and $\theta_{2}=z$, then evidently, $1 \in \mathcal{H}\left(\theta_{1} \theta_{2}\right)$, whereas $1 \notin \mathcal{H}\left(\theta_{1}\right) \cdot \mathcal{H}\left(z \theta_{2}\right)$. Indeed,

$$
\begin{aligned}
1 \in \mathcal{H}\left(\theta_{1}\right) \cdot \mathcal{H}\left(z \theta_{2}\right) & \Longrightarrow 1 \in \mathcal{H}\left(\theta_{1}\right) \cdot(a+b z) \quad \text { for some } a, b \in \mathbb{C} \\
& \Longrightarrow \frac{1}{a+b z} \in \mathcal{H}\left(\theta_{1}\right) \\
& \Longrightarrow \frac{1}{1-\bar{c} z} \in \mathcal{H}\left(\theta_{1}\right) \quad \text { for some }|c|<1 \\
& \Longrightarrow\left\langle e^{\frac{z+1}{z-1}}, \frac{1}{1-\bar{c} z}\right\rangle=0 \\
& \Longrightarrow e^{\frac{c+1}{c-1}}=0,
\end{aligned}
$$

a contradiction. Hence $1 \notin \mathcal{H}\left(\theta_{1}\right) \cdot \mathcal{H}\left(z \theta_{2}\right)$. 
Lemma 2. If $\theta$ is an inner function then

$$
\mathcal{H}(z \theta)=\{\theta \bar{c}: c \in \mathcal{H}(z \theta)\}=H^{2} \cap\left\{\theta \bar{c}: c \in H^{2}\right\} .
$$

Proof. Evidently, $\mathcal{H}(z \theta) \supseteq\{\theta \bar{c}: c \in \mathcal{H}(z \theta)\}$. Note that

$$
f \in \mathcal{H}(z \theta) \Longrightarrow \overline{z \theta} f \in \overline{H_{0}^{2}} \Longrightarrow f \in z \theta \overline{H_{0}^{2}} \Longrightarrow f \in \theta \overline{H^{2}}
$$

Therefore $f=\theta \bar{c}$ for some $c \in H^{2}$ and hence $c=\theta \bar{f}$. Observe that $\overline{z \theta} c=\overline{z \theta} \theta \bar{f}=\overline{z f} \in \overline{H_{0}^{2}}$. Thus $c \in \mathcal{H}(z \theta)$, which proves the first equality. For the second equality, it suffices to prove that if $\theta \bar{c} \in H^{2}$ then $c \in \mathcal{H}(z \theta)$. This follows at once from the observation:

$$
\theta \bar{c} \in H^{2} \Longrightarrow \bar{z} \bar{\theta} c \in \overline{H_{0}^{2}} \Longrightarrow c \in \mathcal{H}(z \theta)
$$

which completes the proof.

Lemma 3. Let $f=\theta_{1} \theta_{2} \bar{a}$ and $g=\theta_{1} \bar{b}$ for $a \in \mathcal{H}\left(\theta_{1} \theta_{2}\right)$ and $b \in \mathcal{H}\left(\theta_{1}\right)$. Then

$$
\frac{f}{g} \in \mathcal{H}\left(z \theta_{2}\right) \Longleftrightarrow \frac{a}{b} \in \mathcal{H}\left(z \theta_{2}\right) .
$$

Proof. Write $\phi:=\frac{f}{g}$ and $\psi:=\frac{a}{b}$. Observe that $\phi=\frac{\theta_{1} \theta_{2} \bar{a}}{\theta_{1} \bar{b}}=\theta_{2} \bar{\psi}$, which implies that $\psi=\theta_{2} \bar{\phi}$. Note that

$$
\phi \in H^{2} \Longleftrightarrow \theta_{2} \bar{\psi} \in H^{2} \Longleftrightarrow \overline{z \theta_{2}} \psi \in \overline{H_{0}^{2}}
$$

Therefore

$$
\begin{aligned}
\phi \in \mathcal{H}\left(z \theta_{2}\right) & \Longleftrightarrow \phi \in H^{2} \text { and } \overline{z \theta_{2}} \phi \in \overline{H_{0}^{2}} \\
& \Longleftrightarrow \overline{z \theta_{2}} \psi \in \overline{H_{0}^{2}} \text { and } \psi \in H^{2} \\
& \Longleftrightarrow \psi \in \mathcal{H}\left(z \theta_{2}\right) .
\end{aligned}
$$

The following lemma is used in proving the main theorem.

Lemma 4. ([Gu2, Corollary 3.5]) Let $\varphi=\bar{g}+f \in L^{\infty}$, where $f=\theta_{1} \theta_{2} \bar{a}$ and $g=\theta_{1} \bar{b}$ for $a \in \mathcal{H}\left(\theta_{1} \theta_{2}\right)$ and $b \in \mathcal{H}\left(\theta_{1}\right)$. Let

$$
\varphi^{\prime}:=\overline{\theta_{1} \bar{b}}+\theta_{1} \overline{P_{\mathcal{H}\left(\theta_{1}\right)}(a)}
$$

Then $T_{\varphi}$ is hyponormal if and only if $T_{\varphi^{\prime}}$ is. Moreover, $\mathcal{E}(\varphi)=\left\{k \theta_{2}: k \in \mathcal{E}\left(\varphi^{\prime}\right)\right\}$.

Lemma 4 says that when we study the hyponormality of Toeplitz operators $T_{\varphi}$ with bounded type symbols $\varphi$, we may assume that the symbol $\varphi=\bar{g}+f \in L^{\infty}$ is of the form

$$
f=\theta \bar{a} \quad \text { and } \quad g=\theta \bar{b}
$$


where $\theta$ is an inner function and $a, b \in \mathcal{H}(\theta)$.

In view of Lemmas 1 and 3 we can introduce a notion of "division" for rational functions. Definition 5. Let $f=\theta_{1} \theta_{2} \bar{a}$ and $g=\theta_{1} \bar{b}$ for $a \in \mathcal{H}\left(\theta_{1} \theta_{2}\right)$ and $b \in \mathcal{H}\left(\theta_{1}\right)$, where the $\theta_{i}$ are finite Blaschke products for $i=1,2$. We shall say that $g$ divides $f$ if $\frac{f}{g} \in \mathcal{H}\left(z \theta_{2}\right)$, or equivalently, $\frac{a}{b} \in \mathcal{H}\left(z \theta_{2}\right)$.

We examine Definition 5 for the cases of polynomials. For example if $f=\sum_{j=1}^{N} a_{j} z^{j}$ and $g=\sum_{j=1}^{n} b_{j} z^{j}$, put

$$
\theta_{1}:=z^{n}, \quad \theta_{2}:=z^{N-n}, \quad a:=\sum_{j=0}^{N-1} \overline{a_{N-j}} z^{j}, \quad \text { and } \quad b:=\sum_{j=0}^{n-1} \overline{b_{n-j}} z^{j} .
$$

Then

$$
f=\theta_{1} \theta_{2} \bar{a}, \quad g=\theta_{1} \bar{b}, \quad a \in \mathcal{H}\left(\theta_{1} \theta_{2}\right), \quad \text { and } \quad b \in \mathcal{H}\left(\theta_{1}\right) .
$$

Thus $g$ divides $f$ if and only if $\frac{a}{b} \in \mathcal{H}\left(z \theta_{2}\right)=\mathcal{H}\left(z^{N-n+1}\right)$, i.e., $\frac{a}{b}=\sum_{j=0}^{N-n} c_{j} z^{j} \in$ $\mathcal{H}\left(z^{N-n+1}\right)$ for some $c_{j}(0 \leq j \leq N-n)$. This exactly coincides with the usual concept of division for polynomials.

We then have:

Theorem 6. Let $\varphi=\bar{g}+f \in L^{\infty}$, where $f=\theta_{1} \theta_{2} \bar{a}$ and $g=\theta_{1} \bar{b}$ for $a \in \mathcal{H}\left(\theta_{1} \theta_{2}\right)$ and $b \in \mathcal{H}\left(\theta_{1}\right)$ with finite Blaschke products $\theta_{1}$ and $\theta_{2}$. If $g$ divides $f$ and $\psi:=\frac{a}{b} \in \mathcal{H}\left(z \theta_{2}\right)$ then the following are equivalent:

(i) $T_{\varphi}$ is hyponormal;

(ii) There exists a function $k \in H^{\infty}$ with $\|k\|_{\infty} \leq 1$ such that $k \psi \in 1+\theta_{1} H^{2}$.

(iii) $T_{\zeta}$ is hyponormal, where $\zeta=\bar{\theta}_{1}+\theta_{1} \overline{P_{\mathcal{H}\left(z \theta_{1}\right)}(\psi)}$.

Moreover if $T_{\varphi}$ is hyponormal then $|\psi(\alpha)| \geq 1$ for each zero $\alpha$ of $\theta_{1}$. In particular, if $\theta_{1}=\theta_{2}$ then

$$
T_{\varphi} \text { is hyponormal } \Longleftrightarrow T_{\bar{\theta}_{1}+\theta_{1} \bar{\psi}} \text { is hyponormal. }
$$

Proof. (i) $\Leftrightarrow$ (ii): Let $\varphi^{\prime}:=\theta_{1} \overline{P_{\mathcal{H}\left(\theta_{1}\right)}(a)}+\bar{g}$. Then by Lemma 4 we have that $\mathcal{E}(\varphi)=\left\{k \theta_{2}\right.$ : $\left.k \in \mathcal{E}\left(\varphi^{\prime}\right)\right\}$. Therefore

$$
\begin{aligned}
T_{\varphi} \text { is hyponormal } \Longleftrightarrow \exists k^{\prime} \in \mathcal{E}(\varphi) & \Longleftrightarrow \overline{\theta_{1}} b-k^{\prime} \overline{\theta_{1} \theta_{2}} a \in H^{2} \text { and }\left\|k^{\prime}\right\|_{\infty} \leq 1 \\
& \Longleftrightarrow \overline{\theta_{1}} b-k \overline{\theta_{1}} a \in H^{2} \text { and }\|k\|_{\infty} \leq 1 \quad\left(k^{\prime}=k \theta_{2}\right) \\
& \Longleftrightarrow b(1-k \psi) \in \theta_{1} H^{2} \text { and }\|k\|_{\infty} \leq 1
\end{aligned}
$$

But since the inner parts of $b$ and $\theta_{1}$ are relatively prime and by assumption, $\psi \in H^{2}$, it follows that

$$
\begin{aligned}
k^{\prime} \in \mathcal{E}(\varphi) & \Longleftrightarrow 1-k \psi \in \theta_{1} H^{2} \text { and }\|k\|_{\infty} \leq 1 \\
& \Longleftrightarrow k \psi \in 1+\theta_{1} H^{2} \text { and }\|k\|_{\infty} \leq 1
\end{aligned}
$$


(ii) $\Leftrightarrow$ (iii): Observe that

$$
\begin{aligned}
k \psi \in 1+\theta_{1} H^{2} & \Longleftrightarrow \overline{\theta_{1}}-k \overline{\theta_{1}} \psi \in H^{2} \\
& \Longleftrightarrow \overline{\theta_{1}}-k \overline{\theta_{1} \bar{\psi}} \in H^{2} \\
& \Longleftrightarrow \overline{\theta_{1}}-k \overline{P\left(\theta_{1} \bar{\psi}\right)} \in H^{2} .
\end{aligned}
$$

But since $P\left(\theta_{1} \bar{\psi}\right)=\theta_{1} \overline{P_{\mathcal{H}\left(z \theta_{1}\right)}(\psi)}$, it follows that $T_{\varphi}$ is hyponormal if and only if $T_{\bar{\theta}_{1}+\theta_{1}} \overline{P_{\mathcal{H}\left(z \theta_{1}\right)}(\psi)}$ is hyponormal.

On the other hand, if $T_{\varphi}$ is a hyponormal operator and if $\theta_{1}(\alpha)=0$ then by (ii)

$$
k(\alpha) \psi(\alpha)=1 \Longrightarrow k(\alpha)=\frac{1}{\psi(\alpha)},
$$

which implies that $|\psi(\alpha)| \geq 1$ since $\|k\|_{\infty} \leq 1$. The last assertion (7) follows at once from the observation that if $\theta_{1}=\theta_{2}$ then $P_{\mathcal{H}\left(z \theta_{1}\right)}(\psi)=\psi$.

Example 7. Let

$$
\varphi=\bar{z} \prod_{j=1}^{8}\left(\overline{B_{j}}-\frac{1}{2}\right)+\frac{8}{7} z \prod_{j=1}^{9}\left(B_{j}-\frac{1}{2}\right),
$$

where $B_{j}(z)=\frac{z+\frac{1}{2}}{1+\frac{1}{2} z}(1 \leq j \leq 9)$. Then $T_{\varphi}$ is not hyponormal.

Proof. Observe that

$$
g=z \prod_{j=1}^{8} B_{j} \cdot \prod_{j=1}^{8}\left(1-\frac{1}{2} \overline{B_{j}}\right) \quad \text { and } \quad f=\frac{8}{7} z \prod_{j=1}^{9} B_{j} \cdot \prod_{j=1}^{9}\left(1-\frac{1}{2} \overline{B_{j}}\right) .
$$

Then

$$
\psi(z)=\frac{8}{7}\left(1-\frac{1}{2} B_{9}(z)\right)
$$

Thus $\psi(0)=\frac{6}{7}<1$. Therefore by Theorem $6, T_{\varphi}$ is not hyponormal.

Example 8. Let

$$
\varphi=\bar{z} \prod_{j=1}^{8}\left(\overline{B_{j}}-\frac{1}{2}\right)+4 z \prod_{j=1}^{9}\left(B_{j}-\frac{1}{2}\right)
$$

where $B_{j}(z)=\frac{z+\frac{1}{2}}{1+\frac{1}{2} z}(1 \leq j \leq 9)$. Then $T_{\varphi}$ is hyponormal.

Proof. Observe that

$$
g=z \prod_{j=1}^{8} B_{j} \cdot \prod_{j=1}^{8}\left(1-\frac{1}{2} \overline{B_{j}}\right) \quad \text { and } \quad f=z \prod_{j=1}^{9} B_{j} \cdot 4 \prod_{j=1}^{9}\left(1-\frac{1}{2} \overline{B_{j}}\right) .
$$

Then

$$
\psi(z)=4\left(1-\frac{1}{2} B_{9}(z)\right)
$$

Put

$$
k(z):=\frac{1+z \prod_{j=1}^{8} B_{j}(z)}{4\left(1-\frac{1}{2} B_{9}(z)\right)}
$$

Then $\|k\|_{\infty} \leq 1$ and $k(z) \cdot \psi(z)=1+z \prod_{j=1}^{8} B_{j}(z)$. Therefore by Theorem 6 (ii), $T_{\varphi}$ is hyponormal. 
Corollary 9. Let $\varphi=\bar{g}+f \in L^{\infty}$, where $f=\theta_{1} \theta_{2} \bar{a}$ and $g=\theta_{1} \bar{b}$ for $a \in \mathcal{H}\left(\theta_{1} \theta_{2}\right)$ and $b \in \mathcal{H}\left(\theta_{1}\right)$ with finite Blaschke products $\theta_{1}$ and $\theta_{2}$. If $\psi:=\frac{f}{g} \in \mathcal{H}\left(z \theta_{2}\right)$ and $|a(\alpha)|=|b(\alpha)|$ for some zero $\alpha$ of $\theta_{1}$ then

$$
T_{\varphi} \text { is hyponormal } \Longleftrightarrow \mathcal{E}(\varphi)=\left\{\frac{b(\alpha)}{a(\alpha)} \theta_{2}\right\}
$$

Proof. By Theorem 6 we have that

$$
T_{\varphi} \text { hyponormal } \Longleftrightarrow \exists k \in H^{\infty} \text { with }\|k\|_{\infty} \leq 1 \text { such that } k \psi \in 1+\theta_{1} H^{2} .
$$

Thus if $T_{\varphi}$ is hyponormal then $k(\alpha) \psi(\alpha)=1$ for each zero $\alpha$ of $\theta_{1}$, so that $k(\alpha)=\frac{b(\alpha)}{a(\alpha)}$. Therefore by the maximum modulus principle, $k(z)=\frac{b(\alpha)}{a(\alpha)}$, and hence $\mathcal{E}(\varphi)=\left\{\frac{b(\alpha)}{a(\alpha)} \theta_{2}\right\}$.

In Theorem 6 , the conditions " $g$ divides $f$ " and " $\theta_{1}=\theta_{2}$ " seem to be too rigid. However the following theorem shows that we may assume, without loss of generality, that $g$ divides $f$ and moreover $\theta_{1}=\theta_{2}$ when we consider the hyponormality of $T_{\varphi}$.

Theorem 10. Let $\varphi=\bar{g}+f \in L^{\infty}$, where $f=\theta_{1} \theta_{2} \bar{a}$ and $g=\theta_{1} \bar{b}$ for $a \in \mathcal{H}\left(\theta_{1} \theta_{2}\right)$ and $b \in \mathcal{H}\left(\theta_{1}\right)$ with a finite Blaschke product $\theta_{1}$. If we let

$$
f_{c}=\theta_{1}^{2} \overline{P_{\mathcal{H}\left(\theta_{1}\right)}(a)}+\theta_{1} \bar{c} \quad \text { for } c \in \mathcal{H}\left(z \theta_{1}\right)
$$

and put $\varphi_{c}:=\bar{g}+f_{c}$, then we have:

(i) $T_{\varphi}$ is hyponormal if and only if $T_{\varphi_{c}}$ is;

(ii) $g$ divides $f_{c}$ for some $c \in \mathcal{H}\left(z \theta_{1}\right)$.

Proof. Write

$$
\theta_{1}=e^{i \xi} \prod_{i=1}^{n} B_{i}^{n_{i}}
$$

where

$$
B_{i}(z):=\frac{z-\alpha_{i}}{1-\overline{\alpha_{i}} z}, \quad\left(\left|\alpha_{i}\right|<1\right), \quad n_{i} \geq 1, \quad \text { and } \quad \sum_{i=1}^{n} n_{i}=d .
$$

Put $a_{0}=P_{\mathcal{H}\left(\theta_{1}\right)}(a)$. Then for each $c \in \mathcal{H}\left(z \theta_{1}\right), f_{c}=\theta_{1}^{2}\left(\overline{a_{0}+\theta_{1} c}\right)$ and $P_{\mathcal{H}\left(\theta_{1}\right)}\left(a_{0}+\theta_{1} c\right)=a_{0}$. Thus by Lemma $4, T_{\varphi}$ is hyponormal if and only if $T_{\varphi_{c}}$ is. To prove (ii) observe that by Lemma 2,

$$
\begin{aligned}
\phi_{c}:=\frac{f_{c}}{g} \in \mathcal{H}\left(z \theta_{1}\right) & \Longleftrightarrow \theta_{1}^{2} \overline{a_{0}}+\theta_{1} \bar{c} \in\left(\theta_{1} \bar{b}\right) \cdot \mathcal{H}\left(z \theta_{1}\right) \text { for some } c \in \mathcal{H}\left(z \theta_{1}\right) \\
& \Longleftrightarrow \theta_{1}^{2} \overline{a_{0}}+\theta_{1} \bar{c} \in\left(\theta_{1} \bar{b}\right) \cdot \mathcal{H}\left(z \theta_{1}\right) \text { for some } c \in H^{2} \\
& \Longleftrightarrow \theta_{1}^{2}\left(\overline{a_{0}+\theta_{1} c}\right)=\theta_{1} \bar{b} \theta_{1} \bar{k} \text { for some } \theta_{1} \bar{k} \in H^{2} \\
& \Longleftrightarrow a_{0}+\theta_{1} c=b k \text { for some } k \in \mathcal{H}\left(z \theta_{1}\right) \\
& \Longleftrightarrow a_{0}-b k \in \theta_{1} H^{2} \text { for some } k \in \mathcal{H}\left(z \theta_{1}\right)
\end{aligned}
$$


Note that $\theta_{1}^{(n)}\left(\alpha_{i}\right)=0$ for all $0 \leq n<n_{i}$. Thus the condition (8) is equivalent to the following equation: for all $1 \leq i \leq n$,

$$
\left[\begin{array}{c}
k_{i, 0} \\
k_{i, 1} \\
k_{i, 2} \\
\vdots \\
k_{i, n_{i}-2} \\
k_{i, n_{i}-1}
\end{array}\right]=\left[\begin{array}{cccccc}
b_{i, 0} & 0 & 0 & 0 & \ldots & 0 \\
b_{i, 1} & b_{i, 0} & 0 & 0 & \ldots & 0 \\
b_{i, 2} & b_{i, 1} & b_{i, 0} & 0 & \ldots & 0 \\
\vdots & \ddots & \ddots & \ddots & \ddots & \vdots \\
b_{i, n_{i}-2} & b_{i, n_{i}-3} & \ddots & \ddots & b_{i, 0} & 0 \\
b_{i, n_{i}-1} & b_{i, n_{i}-2} & \ldots & b_{i, 2} & b_{i, 1} & a_{i, 0}
\end{array}\right]^{-1}\left[\begin{array}{c}
a_{i, 0} \\
a_{i, 1} \\
a_{i, 2} \\
\vdots \\
a_{i, n_{i}-2} \\
a_{i, n_{i}-1}
\end{array}\right],
$$

where

$$
k_{i, j}:=\frac{k^{(j)}\left(\alpha_{i}\right)}{j !}, \quad a_{i, j}:=\frac{a_{0}^{(j)}\left(\alpha_{i}\right)}{j !} \quad \text { and } \quad b_{i, j}:=\frac{b^{(j)}\left(\alpha_{i}\right)}{j !} .
$$

Thus there exists a function $k \in H^{\infty}$ satisfying (8) if and only if there exists a function $k \in H^{\infty}$ for which

$$
\frac{k^{(j)}\left(\alpha_{i}\right)}{j !}=k_{i, j} \quad\left(1 \leq i \leq n, 0 \leq j<n_{i}\right)
$$

where the $k_{i, j}$ are determined by the equation (9). If in addition $\|k\|_{\infty} \leq 1$ is required then this is exactly the classical Hermite-Fejér interpolation problem. But it is well known that there always exists a polynomial $k$ satisfying (10) (cf. $[\mathrm{FF}]$ ). We now find such a funtion $k$ in $\mathcal{H}\left(z \theta_{1}\right)$. Observe that

$$
\begin{aligned}
a_{0}-b k \in \theta_{1} H^{2} & \Longleftrightarrow P_{\mathcal{H}\left(\theta_{1}\right)}\left(a_{0}-b k\right)=0 \\
& \Longleftrightarrow a_{0}-P_{\mathcal{H}\left(\theta_{1}\right)}(b k)=0 \\
& \Longleftrightarrow a_{0}-P_{\mathcal{H}\left(\theta_{1}\right)}\left(b P_{\mathcal{H}\left(\theta_{1}\right)} k\right)=0 \\
& \Longleftrightarrow P_{\mathcal{H}\left(\theta_{1}\right)}\left(a_{0}-b P_{\mathcal{H}\left(\theta_{1}\right)} k\right)=0 \\
& \Longleftrightarrow a_{0}-b P_{\mathcal{H}\left(\theta_{1}\right)} k \in \theta_{1} H^{2} .
\end{aligned}
$$

If we put $k_{1}:=P_{\mathcal{H}\left(\theta_{1}\right)} k$ then $k_{1}$ satisfies $(8)$ and $k_{1} \in \mathcal{H}\left(z \theta_{1}\right)$. This completes the proof.

\section{REFERENCES}

[Ab] M. B. Abrahamse, Subnormal Toeplitz operators and function of bounded type, Duke Math. J. 43 (1976), 597-604.

[BH] A. Brown and P. R. Halmos, Algebric properties of Toeplitz operators, J. Reine Angew. Math. 213 (1963/64), 89-102.

[Co] C. Cowen, Hyponormality of Toeplitz operators, Proc. Amer. Math. Soc. 103 (1988), 809-812.

[FF] C. Foias and A. Frazo, The commutant lifting approach to interpolation problem, Operator Theory: Adv. Appl. vol 44, Birkhäuser, Boston, 1993.

[Gu1] C. Gu, A generalization of Cowen's characterization of hyponormal Toeplitz operators, J. Funct. Anal. 124 (1994), 135-148.

[Gu2] C. Gu, On a class of jointly hyponormal Toeplitz operators, Trans. Amer. Math. Soc. 354 (2002), 3275-3298.

[GS] C. Gu and J. E. Shapiro, Kernels of Hankel operators and hyponormality of Toeplitz operators, Math. Ann. 319 (2001), 553-572. 
[HL] I. S. Hwang and W. Y. Lee, Hyponormality of trigonometric Toeplitz operators, Trans. Amer. Math. Soc. 354 (2002), 2461-2474.

[NT] T. Nakazi and K. Takahashi, Hyponormal Toeplitz operators and extremal problems of Hardy spaces, Trans. Amer. Math. Soc. 338 (1993), 753-769.

[Zhu] K. Zhu, Hyponormal Toeplitz operators with polynomial symbols, Integral Equations Operator Theory 21 (1995), 376-381. 